\title{
Perspectiva geral da endocrinopatia Síndrome dos Ovários Policísticos: uma revisão narrativa
}

\author{
Overview of Polycystic Ovary Syndrome endocrinopathy: a narrative review
}
Descripción general de la endocrinopatía por Síndrome de Ovario Poliquístico: una revisión narrativa

Laís Assunção Vilefort ${ }^{1 *}$, Laura Vitória de Oliveira Frade ${ }^{1}$, Marcelo Hot Petronilho1, Anna Carolina dos Reis Santos², Igor Henrique Vidal ${ }^{2}$, Júlia Cristina Peres Rodrigues Gomes ${ }^{2}$, Laura Simões Bernardes $^{2}$, Luiza Monteiro dos Santos ${ }^{3}$, Yara Zancanaro ${ }^{4}$, Thainah Pereira Rocha ${ }^{5}$.

\section{RESUMO}

Objetivo: Revisar na literatura científica os aspectos em uma ampla abordagem sobre a Síndrome dos Ovários Policísticos (SOP). Revisão bibliográfica: A SOP é uma endocrinopatia muito comum em mulheres, principalmente na idade reprodutiva. Seu quadro clínico é caracterizado por hiperandrogenismo, disfunção ovulatória e infertilidade. Além disso, está associada a fatores de risco cardiovasculares como obesidade, resistência à insulina, dislipidemias, hipertensão arterial sistêmica, síndrome metabólica e diabetes mellitus tipo 2. Por conta da extensa variabilidade clínica, o diagnóstico da SOP pode ser difícil. O tratamento é, na maior parte das vezes, sintomático e estabelecido de acordo com as principais manifestações clínicas da paciente, o desejo de contracepção ou gestação e a presença de distúrbios metabólicos associados. Considerações finais: A SOP deve ser diagnosticada e tratada preferencialmente por um médico ginecologista obstetra com o intuito de reduzir o risco de complicações associadas à doença e melhorar a qualidade de vida da paciente afetada.

Palavras-chave: Síndrome dos ovários policísticos, Anovulação, Idade fértil.

\begin{abstract}
Objective: Review in the scientific literature the aspects of a broad approach on the Polycystic Ovary Syndrome (PCOS). Bibliography review: PCOS is a very common endocrinopathy in women, especially in reproductive age. Its clinical picture is characterized by hyperandrogenism, ovulatory dysfunction and infertility. In addition, it is associated with cardiovascular risk factors such as obesity, insulin resistance, dyslipidemia, systemic arterial hypertension, metabolic syndrome and type 2 diabetes mellitus. Due to the extensive clinical variability, the diagnosis of PCOS can be difficult. Treatment is, in most cases, symptomatic and established according to the main clinical manifestations of the patient, the desire for contraception or pregnancy, and the presence of associated metabolic disorders. Final considerations: PCOS should be diagnosed and preferably treated by an obstetrician gynecologist in order to reduce the risk of complications associated with the disease and improve the quality of life of the affected patient.
\end{abstract}

Key words: Polycystic ovary syndrome, Anovulation, Childbearing age.

\section{RESUMEN}

Objetivo: Revisar en la literatura científica los aspectos de un abordaje amplio sobre el Síndrome de Ovario Poliquístico (SOP). Revisión bibliográfica: El síndrome de ovario poliquístico es una endocrinopatía muy común en las mujeres, especialmente en la edad reproductiva. Su cuadro clínico se caracteriza por

\footnotetext{
${ }^{1}$ Faculdade de Minas de Belo Horizonte (FAMINAS-BH), Belo Horizonte - MG.

*E-mail: lavilefort@gmail.com

2 Universidade José do Rosário Vellano (UNIFENAS-BH), Belo Horizonte - MG

${ }^{3}$ Centro Universitário de Belo Horizonte (UNI-BH), Belo Horizonte - MG.

${ }^{4}$ Faculdade Evangélica Mackenzie do Paraná (FEMPAR), Curitiba - PR.

${ }^{5}$ Centro Universitário de Caratinga (UNEC), Caratinga - MG.
} 
hiperandrogenismo, disfunción ovulatoria e infertilidad. Además, se asocia con factores de riesgo cardiovascular como obesidad, resistencia a la insulina, dislipidemia, hipertensión arterial sistémica, síndrome metabólico y diabetes mellitus tipo 2. Debido a la amplia variabilidad clínica, el diagnóstico del SOP puede ser difícil. El tratamiento es, en la mayoría de los casos, sintomático y se establece según las principales manifestaciones clínicas de la paciente, el deseo de anticoncepción o embarazo y la presencia de trastornos metabólicos asociados. Consideraciones finales: EI SOP debe ser diagnosticado y tratado preferiblemente por un ginecólogo obstetra para reducir el riesgo de complicaciones asociadas con la enfermedad y mejorar la calidad de vida del paciente afectado.

Palabras clave: Síndrome de ovario poliquístico, Anovulación, Edad fértil.

\section{INTRODUÇÃO}

A Síndrome dos Ovários Policísticos (SOP) é uma endocrinopatia bastante comum no período do menacme. Também conhecida como anovulação crônica por retrocontrole impróprio, acomete pelo menos uma a cada cinco mulheres em idade reprodutiva, podendo variar de acordo com o critério diagnóstico utilizado e a população estudada. O hiperandrogenismo clínico ou bioquímico é a sua principal característica e está associado à presença de ovários policísticos e à disfunção menstrual (AVERSA A, et al., 2020).

A SOP torna-se uma patologia de grande importância não só pelas suas manifestações clínicas, mas também pelas repercussões que pode trazer ao longo da vida à saúde da paciente, como por exemplo, infertilidade, dislipidemia, obesidade, resistência insulínica, Diabetes Mellitus tipo 2 (DMS), diabetes gestacional, maior risco cardiometabólico, redução da qualidade de vida e depressão. Além disso, o custo econômico para tratamento e diagnóstico das diversas morbidades associadas à ela é bem alto. Cerca de quatro bilhões de dólares são gastos nos Estados Unidos anualmente para este fim (RAPERPORT C e HOMBURG R, 2019).

O distúrbio ovariano é estabelecido pelo hiperandrogenismo e por alterações da secreção gonadotrópica, e acaba se manifestando por distúrbios menstruais, hirsutismo e infertilidade. O distúrbio metabólico é reconhecido pela hiperinsulinemia reativa, resistência à insulina, intolerância à glicose e dislipidemia. Sendo esta última marcada pela diminuição de lipoproteínas de alta densidade (HDL-colesterol) e pelo aumento de lipoproteínas de baixa densidade (LDL-colesterol) e triglicerídeos (MINISTÉRIO DA SAÚDE, 2019).

A sua etiologia ainda não é muito bem estabelecida, sendo considerada uma incógnita. Isso se dá pelo fato da possibilidade de ter vários fatores que podem estar envolvidos em sua gênese. Partindo do ponto de que esta é uma doença metabólica, a relação com alterações no metabolismo de lipídios e glicose tem sido alvo de muitos estudos. O foco nesta doença deixou de ser apenas o sistema genital feminino e reprodutor, e passou a ser o organismo de uma forma geral, o que tem gerado o interesse e a preocupação de outros profissionais da saúde sobre essas pacientes (AJMAL N, et al., 2019).

O objetivo desta revisão foi fornecer através de uma revisão na literatura científica uma ampla abordagem sobre a Síndrome dos Ovários Policísticos, incluindo suas manifestações clínicas, diagnóstico e tratamento, além de orientar sobre suas principais complicações.

\section{REVISÃO BIBLIOGRÁFICA}

\section{Epidemiologia da SOP}

Em mulheres com idade reprodutiva, a SOP é a patologia endócrina considerada mais em todo o mundo, afetando cerca de $5 \%$ a $15 \%$ das mulheres, variando de acordo com os critérios diagnósticos. Com base no relatório do workshop do National Institutes of Health (NIH) de 2012, estima-se que cerca de 5 milhões de mulheres em idade reprodutiva são acometidas pela SOP nos Estados Unidos. Para seu diagnóstico e tratamento, calcula-se um custo de aproximadamente 4 bilhões de dólares anualmente para o sistema de saúde, sem incluir o custo das comorbidades graves associadas a ela (LEON LIR, et al., 2021). 


\section{Manifestações clínicas da SOP}

O quadro clínico da SOP envolve queixascomo irregularidades menstruais, hirsutismo, acantose nigrans, obesidade, acne persistente, seborréia, alopecia e hiper-hidrose. O melhor marcador clínico de hiperandrogenismo na adolescência é o quadro de hirsutismo. Isso ocorre devido ao fato de que acne é uma manifestação transitória e com aparecimento típiconessa faixa etária enquanto a alopecia é uma manifestação pouco comum nessa população. Na adolescência, a amenorreia secundária, a oligomenorreia e anovulação crônica são muito frequentes e, cerca de $40 \%$ a $50 \%$ das muheres apresentam ciclos anovulatórios (MINISTÉRIO DA SAÚDE, 2019).

Nessas pacientes, o hiperandrogenismo é caracterizado pela presença de acne do tipo severa que não responde a tratamentos tópicos, por ciclos menstruais irregulares, isto é, com intervalos menores que 21 dias ou maiores que 45 dias e, pela presença de hirsutismo do tipo moderado ou severo. Como essas pacientes possuem maiores riscos de alterações metabólicas, elas aumentam a probabilidade de desenvolverem dislipidemia, sobrepeso, obesidade, pré diabetes, diabetes mellitus tipo 2, apneia obstrutiva do sono, doenças hepáticas não associadas ao etilismo, distúrbios de humor e neoplasias. No que diz respeito à reprodução, mulheres com SOP possuem, quando comparadas à população de uma forma geral, elevado risco de infertilidade e aumentam as taxas de abortamento espontâneo (BELLVER J, et al., 2018).

\section{Fisiopatologia da SOP}

A fisiopatologia desta doença não é muito bem definida e nem única, porém trata-sede um distúrbio metabólico e reprodutivodo tipo heterogêneo, levando a um quadro sindrômico. Em sua etiopatogenia existem diversos fatores implicados, possuindo componentes genéticos envolvidos, distúrbios endócrinos hereditários, fatores metabólicos pré e pós-natais e fatores ambientais como a prática de exercísios físicos e dieta (WITCHEL SF, et al., 2019).

Porém, existem alguns mecanismos endócrinos que estão envolvidos na etiopatogênese da SOP, como por exemplo, a forma de secreção de gonadotrofinas, em que, como característica patognomõnica desta síndrome, tem-seuma secreção aumentadado hormônio luteinizante (LH), levando a uma elevação na amplitude dos pulsos e consequente elevação na produção de androgênios, principalmente de testosterona. As mulheres portadoras de SOP possuem o retrocontrole feito pela progesterona e pelos estrogênios origem ovariana com uma sensibilidade hipotalâmica reduzida (WITCHEL SF, et al., 2019).

De maneira bem intensa ocorrem a ativação e o recrutamento folicular, porém isso ocorre com menor atresia dos folículos em estágios iniciais, o que confere uma morfologia policística ao ovário. A produção anômala de androgênios nessas pacientes parece ter uma influência significativa da insulina e do fator de crescimento semelhante à insulina tipo 1. Sendo assim, resistência à insulina e hiperinsulinemia compensatória são comuns de serem encontradas em portadoras de SOP independentemente da presença ou não de obesidade, sendo a resistência tanto para a ação da insulina no tecido adiposo quanto no músculo estriado (BEDNARSKA S e SIJKA A, 2017).

A produção de androgênios ovarianos possui influência direta no aumento da insulina circulante, uma vez que ela possui nas células da tecauma ação sinérgica ao LH, sendo estimulada a produção de androgênios. A redução da produção da proteína carreadora de androgênios (SHBG) pelo fígado também tem relação direta com a ação da insulina e, somados, esses dois efeitos aumentam a concentração da fração ativa do hormônio, isto é, da testosterona livre (ROSENFIELD RL e EHRMANN DA, 2016).

\section{Diagnóstico da SOP}

Para o diagnóstico de SOP, atualmente, o protocolo mais utilizado é o consenso em que este diagnóstico é determinado pela presença de ao menos dois dos três critérios diagnósticos, desde que sejam excluídas outras comorbidades que também podem cursarm com hiperandrogenismo. Como critérios diagnósticos temse: hiperandrogenismo clínico ou laboratorial, oligoamenorreia, morfologia ultrassonográfica de policistose ovariana. Para adolescentes, esses critérios são mais restritos, sendo a morfologia ovariana excluída, e o hiperandrogenismo implicando exclusivamente na presença de hiperandrogenemia ou hirsutismo. Além disso, 
o diagnóstico quando realizado na adolescência deve ser analisado novamenteoito anos apósa menarca mesmo que preencha esses critérios (BACHELOT A, 2016).

$O$ atraso menstrual com ciclos longos pode ser gerado pela anovulação, porém de maneira normoestrogênicos, uma vez que ocorre o crescimento folicular de forma parcial e, por isso, há infertilidade e oligoamenorreia, sendo um histórico menstrual deste último caso caracterizado como a ausência de menstruação por um período de 90 dias ou mais ou a ocorrência de menos de nove ciclos menstruais em um ano. Além disso, os sinais e sintomas clínicos do hiperandrogenismo podem ser originados por efeito direto dos androgênios sobre os folículos sebáceos e pilosos e, como exemplo de manifestações, tem-se: pele oleosa, acne, hirsutismo, queda de cabelo e, nos casos mais graves, sinais de virilização com alopecia androgênica e clitoromegalia (ROCHA AL, et al., 2019).

A característica morfológica dos ovários deve ser avaliada nos casos em que as manifestações clínicas não estão tão exuberantes. Para isso, a ultrassonografia pélvica bidimensional é a forma mais simples e de mais baixo custo, devendo ser realizada, preferencialmente, de forma transvaginal. Nos casos em que 0 fenótipo não é claramente hiperandrogênico, o diagnóstico diferencial com outras causas de anovulação crônica deve ser realizado, sendo as principais causas as anovulações de origem hipotalâmica, a hiperprolactinemia e a insuficiência ovariana prematura (AJMAL N, et al., 2019).

Em pacientes com SOP, é esperado que os valores de prolactina estejam dentro dos padrões de normalidade. Entretanto, esses valores encontram-se elevados em cerca de $10 \%$ dos casos. Ainda não estão muito bem definidos os mecanismos pelos quais isso, mas a produção de prolactina parece haver maior sensibilidade ao estímulo do hormônio liberador de tireotrofina (TRH), principalmente nas portadoras de SOP com níveis de LH elevados. Já os valores de FSH, em função de uma resposta à elevada produção de inibina pelos folículos antrais em crescimento, possivelmente estarão nas pacientes com SOP bem próximos ao menor valor ainda considerado como referência o que é bem característico da doença (MINISTÉRIO DA SAÚDE, 2019).

Apesar de a característica hormonal dessas pacientes estar baseada em uma hipersecreção de LH, não é necessário que seja dosado esse hormônio, uma vez que esse é um exame com baixo valor preditivo, em que menos de $50 \%$ das mulheres portadoras de SOP apresentam níveis superiores desse hormônio em uma dosagem isolada. Na avaliação dessas pacientes, a dosagem de TSH pode ser complementar, uma vez que cerca de $14 \%$ a $20 \%$ delas apresentam valores aumentados desse hormônio e parece que um pior perfil metabólico nessas pacientes pode estar relacionado a essa alteração (AVERSA A, et al., 2020).

\section{Prevalência da Síndrome Metabólica na SOP}

Em pacientes com SOP há uma prevalência da Síndrome Metabólica (SM) de aproximadamente 33\% a $43 \%$ ajustadas para Índice de Massa Corporal (IMC) e idade, o que representa um valor praticamente duas vezes maior do que em mulheres da população de uma forma geral. A associação entre síndrome metabólica e essa patologia aumenta em aproximadamente sete vezes o risco de desenvolvimento de doença cardiovascular. As alterações presentes na função vascular existentes anteriormente aos eventos podem ser comprovadas através de métodos propedêuticos adequados (FEDERAÇÃO BRASILEIRA DAS ASSOCIAÇÕES DE GINECOLOGIA E OBSTETRÍCIA (FEBRASGO), 2019).

A prevalência da síndrome metabólica pode variar de acordo com a região. Nos Estados Unidos (EUA), por exemplo, são quase $50 \%$ das mulheres com SOP as portadoras de SM. Na Itália, a SM é menos comum nessas pacientes, uma vez que as mulheres com SOP possuem um menor peso médio e triglicerídeos séricos elevados de forma menos frequente que nos EUA, possuindo uma frequência de cerca de duas vezes maior na SOP quando comparada a população normal. De uma forma geral, a SM é diagnosticada em mulheres com SOP a partir da terceira década de vida, com uma prevalência que se aproxima daquela em mulheres da população geral próximo aos 50 e 60 anos de idade. Cerca de $23 \%$ dessas mulheres possuem diagnóstico de DM antes dos 19 anos, aproximadamente $45 \%$ delas apresentam entre 20 e 29 anos e $53 \%$ entre 30 e 39 anos. Entre mulheres com valores mais elevados de IMC e insulina, a SM e seus componentes individuais são muito mais comuns (FEBRASGO, 2019). 


\section{Tratamento da SOP}

Na SOP, o tratamento é sintomático e deve ser direcionado de acordo com as manifestações clínicas de cada paciente, de acordo com a presença de distúrbios metabólicos associados e com o desejo de contracepção ou gestação da mesma. Todas as pacientes devem ser contextualizadas sobre o fato de que esta é uma enfermidade crônica, possuindo um seguimento médico e tratamento por longo prazo. O principal objetivo terapêutico é regularizar o ciclo menstrual, oferecer contracepção para as mulheres que não desejam engravidar, induzir a ovulação nas mulheres que desejam gestação, reduzir os sintomas de hiperandrogenismo, prevenir a hiperplasia e câncer de endométrio e diminuir as anormalidades metabólicas, como o risco de doenças cardiovasculares ou DM2 (BEDNARSKA S e SIJKA A, 2017).

Nos casos de mulheres com SOP que possuem sobrepeso ou obesidade já instalada, com o objetivo sendo a redução de peso, a primeira linha de tratamento é a reeducação alimentar, com dieta hipocalórica, aumento de gordura mono e poli-insaturada, diminuição da ingesta de gordura saturada, e aumento do consumo de fibras, frutas, cereais e vegetais. Além disso, a prática de exercício físico é recomendada de forma regular de intensidade variando entre moderada a intensa, por pelo menos cinco dias da semana, com uma duração dos exercícios de, no mínimo, 30 minutos (NICOLAIDES NC, et al., 2020).

Uma conduta fundamental, principalmente para as mulheres com diagnóstico de obesidade central é o incentivo a modificações do estilo de vida, com prática de exercício físico de forma regular associada a medidas dietéticas. Em um período de 6 a 12 meses deve ocorrer uma redução de cerca de 7 a $10 \%$ do peso corporal, sendo a meta principal uma perda de aproximadamente 10 a $20 \%$. Dessa forma, na maior parte dos casos o ciclo menstrual consegue ser regulado e a ovulação espontânea iniciada, bem como uma melhora na resistência à insulina, ao quadro de dislipidemia, à melhora na qualidade de vida e redução de quadros de DM2 e depressão (BELLVER J, et al., 2018).

Em casos de pacientes com SOP com intolerância à glicose ou DM2 está indicado o uso de metformina, sendo recomendada doses entre 500 e $1700 \mathrm{mg} / \mathrm{dia}$, sendo a dose inicial de $500 \mathrm{mg} /$ dia junto às refeições, aumentando de forma gradativa. Como resultado, os efeitos adversos são minimizados, sendo eles: flatulência, diarreia, dor abdominal, náusea e paladar metálico. Há estudos que mostram que a vitamina B12 e o ácido fólico e a vitamina B12 podem minimizar estes efeitos colaterais (LOUWERS YV e LAVEN JSE, 2020).

E casos de pacientes com SOP que não desejam engravidar, o tratamento padrão mais conveniente e considerado de primeira linha é baseado no uso de métodos contraceptivos hormonais, principalmente os Contraceptivos Hormonais Orais Combinados (CHOCs), responsáveis por melhorar o hirsutismo, reduzir acne e regularizar o ciclo menstrual. Os hormônios contidos nos CHOCs diminuem a produção dos androgênios ovarianos por consequência de uma supressão de LH (NICOLAIDES NC, et al., 2020).

A fração de estrogênio contido na pílula estimula a produção de SHBG por via hepática, levando a um aumento na ligação androgênica sérica e redução das concentrações de androgênios livres circulantes, assim como a sua bioatividade. Esses contraceptivos ainda apresentam um efeito no endométrio do tipo antiproliferativo. Outros métodos hormonais também podem ser utilizados, como os combinados (adesivo, injetável mensal e anel vaginal) ou até mesmo os que são compostos apenas de progesterona (via oral, injetável trimestral, dispositivo intrauterino e implante subdérmico) (BEDNARSKA S e SIJKA A, 2017).

Em todas as pacientes deve ser realizada a triagem de contraindicações para anticoncepcionais hormonais, sendo consideradas como contra-indicações absolutas mulheres com 35 anos ou mais com carga tabágica superior a 15 cigarros por dia, diabetes não controlada com doença vascular periférica grave ou hipertensas descompensadas com PA superior a 160x100 $\mathrm{mmHg}$. As pacientes diabéticas que e não possuem complicações vasculares não são consideradas contra-indicação ao uso de anticoncepcionais hormonais (LOUWERS YV e LAVEN JSE, 2020).

Em mulheres com SOP que não são obesas e possuem o desejo de engravidar, o tratamento de primeira linha é o Citrato de Clomifeno (CC), devendo ser utilizado nas doses de 50 a $150 \mathrm{mg}$ entre os $3^{\circ}$ e $7^{\circ}$ dias por 4 ciclos. As ondas de calor são os efeitos colaterais mais comuns. Esse medicamento é um modulador de 
receptor de estrogênio, parcialmente seletivo, com efeito antiestrogênico, isto é, induz uma mudança na frequência e liberação em pulsos do $\mathrm{GnRH}$ levando a um aumento do $\mathrm{FSH}$, culminando em desenvolvimento folicular e produção de estradiol. Sendo assim, em cerca de 75 a $80 \%$ dos casos ocorre a ovulação e a gestação é alcançada em 35 a 40\% dos casos (BEDNARSKA S e SIJKA A, 2017).

O Letrozol, um inibidor da aromatase, possui estudos que mostram ser mais eficaz CC para a indução da ovulação em casos de mulheres obesas com SOP. Porém, além das ondas de calor, a fadiga também é um dos seus efeitos colaterais. Por sua vez, para a indução da ovulação em pacientes com SOP e chance duas vezes maior de gravidez no primeiro ciclo, o uso do hormônio folículo estimulante recombinante (FSHr) em baixa dose é mais eficaz que o (TEEDE HJ, et al., 2018).

\section{Complicações da SOP}

Em mulheres com SOP, o câncer ginecológico mais frequentemente encontrado é o de endométrio. Como os cânceres de mama e ovário não parecem ser mais tão prevalentes em doentes com SOP, o rastreamento para neoplasia de mama não difere entre a população geral e as mulheres com SOP, ele pode variar de acordo com a idade e outros fatores de risco adicionais associados a esta neoplasia. O câncer de endométrio, por sua vez, é cerca de três vezes mais prevalente nas mulheres com anovulação crônica. Embora a presença de SOP não esteja muito associada a um risco aumentado para neoplasia de ovário, a amenorréia ou a oligomenorreia podem apresentar relação com o tipo histopatológico do câncer ovariano (RAPERPORT C e HOMBURG R, 2019).

As mulheres com SOP apresentam prevalências mais elevadas de fatores de risco para doenças cardiovasculares. Uma considerável parcela destas pacientes possui resistência à insulina e apresentam concentrações elevadas de marcadores inflamatórios. Além disso, quando comparadas às mulheres dem diagnóstico de SOP, elas possuem maior probabilidade de apresentar algumas comorbidades como hipertensão arterial, diabetes, dislipidemia, síndrome metabólica, alterações angiográficas, risco elevado de infarto agudo do miocárdio, acidente vascular encefálico e doença arterial coronariana. Existem também algumas evidências de fontes confiáveis que associam o maior risco de eventos tromboembólicos à presença de SOP (BELLVER J, et al., 2018).

\section{CONSIDERAÇÕE FINAIS}

A SOP é uma doença endócrino-metabólica que afeta inúmeras mulheres em idade reprodutiva. Dentre as principais manifestações da doença estão hiperandrogenismo, disfunção ovulatória e infertilidade. A fisiopatologia ainda não é bem definida, mas sabe-se que a hipersecreção do hormônio luteinizante é ponto central da síndrome. O diagnóstico é feito preferencialmente pelo médico ginecologista baseado em critérios clínico/laboratoriais e a doença poderá ser classificada em até quatro fenótipos distintos. O tratamento é realizado de acordo com a clínica apresentada pela paciente e os dois medicamentos mais utilizados são os anticoncepcionais orais combinados e a metformina. Dentre as complicações desta síndrome estão o maior risco de desenvolvimento de câncer ginecológico e aumento do risco cardiovascular global.

\section{REFERÊNCIAS}

1. AJMAL N, et al. Polycystic ovary syndrome (PCOS) and genetic predisposition: A review article. European Journal of Obstetrics \& Gynecology and Reproductive Biology: X, 2019; 3: 100060.

2. AVERSA A, et al. Fundamental Concepts and Novel Aspects of Polycystic Ovarian Syndrome: Expert Consensus Resolutions. Frontiers in Endocrinology, 2020; 11: 516.

3. BACHELOT A. Polycystic ovarian syndrome: clinical and biological diagnosis. Annales de Biologie Clinique, 2016; 74(6): 661-667.

4. BEDNARSKA S, SIJKA A. The pathogenesis and treatment of polycystic ovary syndrome: What's new? Advances in Clinical and Experimental Medicine, 2017; 26(2): 359-367.

5. BELLVER J, et al. Polycystic ovary syndrome throughout a woman's life. The Journal of Assisted Reproduction and Genetics, 2018; 35(1): 25-39.

6. CONCHA FC, et al. Epigenetics of polycystic ovary syndrome. Revista Médica do Chile, 2017; 145(7): $907-915$. 
7. FEDERAÇÃO BRASILEIRA DAS ASSOCIAÇÕES DE GINECOLOGIA E OBSTETRÍCIA (FEBRASGO). SOP Síndrome dos ovários policísticos Repercussões metabólicas de uma doença intrigante. Federação Brasileira das Associações de Ginecologia e Obstetrícia, 2019; 47(9). Disponível em: https://www.febrasgo.org.br/media/k2/attachments/Vol.Z47ZnZ9Z-Z2019.pdf. Acesso em: 01 out 2021.

8. LEON LIR, et al. Polycystic Ovarian Disease. StatPearls Publishing, 2021; 1(2): 1-5.

9. LOUWERS YV, LAVEN JSE. Characteristics of polycystic ovary syndrome throughout life. Therapeutic Advances in Reproductive Health, 2020; 14: 2633494120911038.

10. MINISTÉRIO DA SAÚDE. Protocolo clínico e diretrizes terapêuticas síndrome de ovários policísticos. Secretaria De Atenção Especializada À Saúde Secretaria De Ciência, Tecnologia E Insumos Estratégicos, 2019. Disponível em: https://antigo.saude.gov.br/images/pdf/2019/julho/11/Ovarios-Policisticos-julho2019.pdf. Acesso em: 01 out 2021.

11. NICOLAIDES NC, et al. Polycystic ovarian syndrome in adolescents: From diagnostic criteria to therapeutic management. Acta Biomedica Brasiliensia, 2020; 91(3): e2020085.

12. RAPERPORT C, HOMBURG R. The Source of Polycystic Ovarian Syndrome. Clinical Medicine Insights: Reproductive Health, 2019; 13: 1179558119871467.

13. ROCHA AL, et al. Recent advances in the understanding and management of polycystic ovary syndrome. F1000Research, 2019; 8: F1000 Faculty Rev-565.

14. ROSENFIELD RL, EHRMANN DA. The Pathogenesis of Polycystic Ovary Syndrome (PCOS): The Hypothesis of PCOS as Functional Ovarian Hyperandrogenism Revisited. Endocrine Reviews, 2016; 37(5): 467-520.

15. TEEDE HJ, et al. Recommendations from the international evidence-based guideline for the assessment and management of polycystic ovary syndrome. Fertility and Sterility, 2018; 110(3): 364-379.

16. WITCHEL SF, et al. Polycystic Ovary Syndrome: Pathophysiology, Presentation, and Treatment With Emphasis on Adolescent Girls. Journal of the Endocrine Society, 2019; 3(8): 1545-1573. 